\title{
Distal Ulnar Metaphyseal Wedge Osteotomy for Ulnar Abutment Syndrome
}

\author{
Shunsuke Nishimoto, MD, PhD ${ }^{4}$ Takeshi Yoshida, MD, PhD ${ }^{5}$ \\ ${ }^{1}$ Department of Orthopaedic Surgery, Kyouritsu Hospital, Kawanishi- \\ shi, hyogo, Japan \\ 2 Osaka Yukioka College of Health Science, Yukioka Hospital Hand \\ Center, Osaka-shi, Osaka, Japan \\ ${ }^{3}$ Department of Orthopaedic Surgery, Yukioka Hospital, Osaka, \\ Osaka Prefecture, Japan \\ ${ }^{4}$ Department of Orthopaedic Surgery, Kansai Rosai Hospital, \\ Amagasaki-shi, Hyogo, Japan \\ ${ }^{5}$ Yoshida Orthopaedic Clinic, Itami-shi, Hyogo, Japan
}

Nobuyuki Kubo ${ }^{1}$ Hisao Moritomo, MD, $\mathrm{PhD}^{2}$ Sayuri Arimitsu, MD, PhD 3

J Wrist Surg 2019;8:352-359.

Address for correspondence Hisao Moritomo, MD, PhD, Osaka Yukioka College of Health Science, Yukioka Hospital Hand Center, Osaka-shi, Osaka, Japan (e-mail: hisao-moritomo@yukioka-u.ac.jp).

\section{Abstract \\ Keywords \\ - TFCC \\ - ulnocarpal impaction syndrome \\ - ulnar shortening \\ - nonunion \\ - ulnar abutment syndrome}

Background Ulnar shortening osteotomy of the diaphysis is a common and effective surgical procedure for ulnar abutment syndrome. However, this procedure has some disadvantages, such as a long period until union and a relatively high nonunion rate. To overcome these disadvantages, we have developed distal ulnar metaphyseal wedge osteotomy. The purpose of this article is to describe the technique and to report its clinical results. Patients and Methods Distal ulnar metaphyseal wedge osteotomy consists of resection of the wedge fragment at the distal ulnar metaphysis, compressing the distal fragment of the ulna toward the radial-proximal direction and fixation with a Herbert type headless screw. We performed this procedure for 58 patients with ulnar abutment syndrome, and the clinical data of 43 patients who were followed for $>6$ months were analyzed. We evaluated range of motion, grip strength, and HAND20 which is a validated subjective scoring system in Japan.

Results All patients experienced relief from their ulnar wrist pain, and bone union was achieved within an average of 2.6 months. The range of dorsiflexion improved from $63^{\circ}$ preoperatively to $69^{\circ}$ postoperatively, grip strength compared with the contralateral hand improved from $77 \%$ preoperatively to $87 \%$ postoperatively, and HAND20 improved from 41.3 points preoperatively to 22.4 points postoperatively.

Discussion This procedure has advantages especially in early bone union. This procedure should be taken into consideration as one of the options to treat ulnar abutment syndrome.
Ulnar abutment syndrome is known as a cause of ulnar wrist pain that is difficult to manage. Ulnar shortening osteotomy of the diaphysis is the most common surgical procedure for ulnar abutment syndrome. This procedure was first reported by Milch in 1941, ${ }^{1}$ and many surgeons later reported excellent results. ${ }^{2-9}$ However, the diaphyseal osteotomy has some disadvantages, including a long period until union, with

received

October 23, 2017

accepted after revision

November 26, 2018

published online

February 6, 2019 previous studies reporting 21 to 28 weeks for bony union, ${ }^{2,3}$ a relatively higher nonunion rate, and refracture at the osteotomy site could occur after plate removal. ${ }^{6}$ Moreover, plate irritation often occurs, for which plate removal is required.

Another common procedure for ulnar abutment syndrome is wafer resection of the distal articular surface of
Copyright $\odot 2019$ by Thieme Medical Publishers, Inc., 333 Seventh Avenue, New York, NY 10001, USA. Tel: +1(212) 584-4662.
DOI https://doi.org/ 10.1055/s-0038-1677494. ISSN 2163-3916. 
the ulnar head, which was first described by Feldon et al in $1992 .^{10}$ Although it is a simple and easy procedure, it is arguable whether this operation should be performed for young patients because of the possible risk of future degeneration due to a cartilage defect.

Distal ulnar metaphyseal wedge osteotomy was first reported by Kitano et al in $1993 .^{11}$ In this operation, a wedge-shaped bone block of the distal ulnar metaphysis was resected, and the ulnar head was rotated to the radial-proximal side and fixed with a headless compression screw. In this procedure, a screw was inserted from the distal end of the ulnar head toward the intramedullary region; therefore, screw fixation was not very rigid, so that bony union tended to be delayed in some cases with osteoporosis. To overcome this problem, Yoshida et al modified the procedure to penetrate the screw proximally through the cortex (modified Kitano's method, - Fig. 1) and reported better clinical results in $1998 .^{12}$ However, their results were reported only in the Japanese literature. The purpose of this article is to describe the technique of the modified Kitano's method and to report its clinical results.

\section{Surgical Technique}

Under general or regional axillary anesthesia, the patient is positioned supine on the operating table with the shoulder abducted at $90^{\circ}$ and the forearm pronated on a hand table. A tourniquet is placed on the upper arm.

A 3-cm dorsal longitudinal or curved skin incision is made just on the fifth compartment ( - Fig. 2A). Incising the extensor retinaculum, the extensor digiti minimi is retracted radially, and the capsule of the distal radioulnar joint (DRUJ) is exposed. After the capsule of the DRUJ is incised, the ulnar head is exposed (-Fig. 2B). The proximal surface of the triangular fibrocartilage complex (TFCC) can be observed, usually showing fibrillation of the disc and the radioulnar ligament, and often with perforation of the disc. It is necessary to confirm that the foveal insertion of the TFCC is intact (-Fig. 2C). Next, it is recommended that the sixth compartment of the extensor retinaculum be incised to prevent tendinitis of the extensor carpi ulnaris (ECU) tendon by radial translation of the ulnar styloid after osteotomy. The subsheath of the ECU tendon that is attached to the distal ulna and considered as a stabilizer of the DRUJ must be preserved to avoid dislocation of the ECU tendon (-Fig. 2D). ${ }^{13}$

Wedge osteotomy of the ulnar metaphysis is performed using an oscillating saw (- Fig. 2E, F). If 3-mm shortening is performed, the $3-\mathrm{mm}$ base of the wedge is placed on the side opposite the ulnar styloid. Periosteal continuity of the ulnar aspect of the osteotomy site should be maintained for rigid fixation. If it is cut completely, the ulnar head becomes unstable. After resection of the wedge fragment, the distal fragment of the ulna is compressed toward the proximal direction (-Fig. 2G). A Herbert type headless screw is inserted from the center of the articular surface of the ulnar head to the ulnar side cortex over the osteotomy site (-Fig. $\mathbf{2 H}$ ). The osteotomy site is fixed rigidly, and ulnar shortening is completed. The distal tip of the screw must be buried under the cartilage of the articular surface (-Fig. 21). When inserting the screw, temporary pinning is useful to prevent rotation of the ulnar head.

As postoperative therapy, the wrist is immobilized for 3 weeks after operation by a splint in the shape of sugar tongs (-Fig. 3) to prevent pronation and supination of the wrist. After 3 weeks, motion exercise of the wrist is initiated. A removable short arm orthosis is useful to avoid overstress

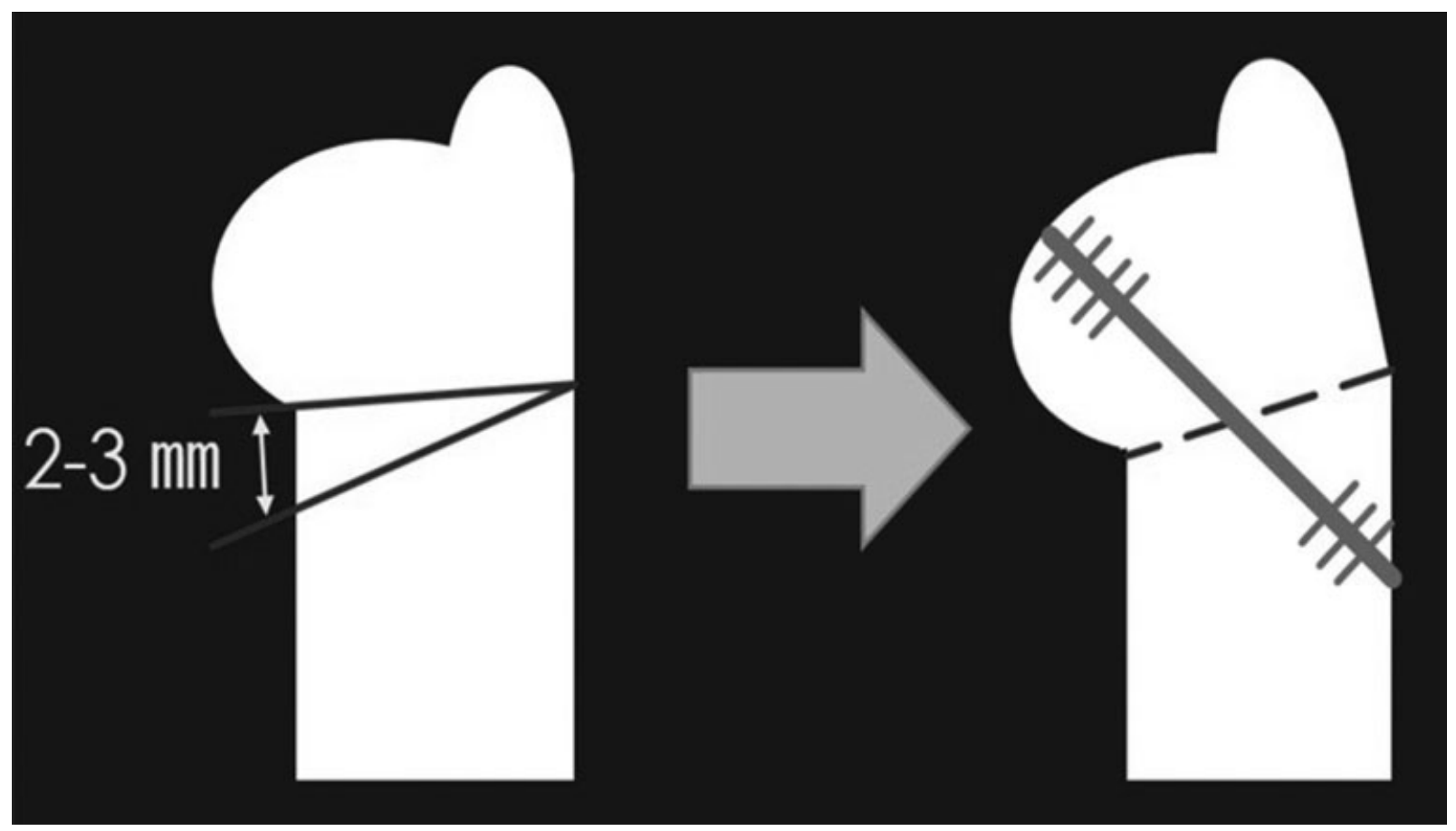

Fig. 1 Schema of distal ulnar metaphyseal wedge osteotomy. 

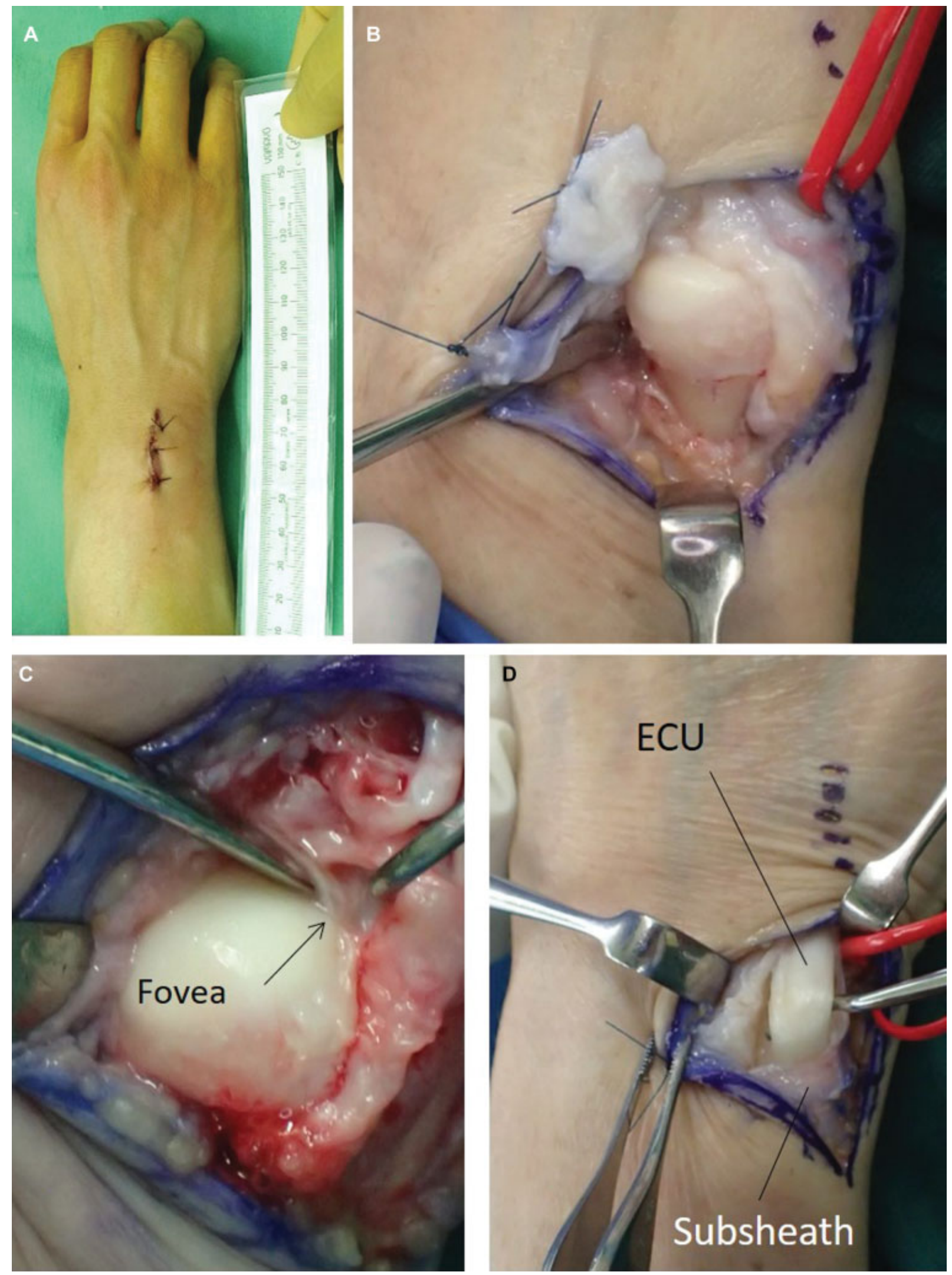

Fig. 2 (A) Skin incision, (B) opened joint, (C) check the fovea, (D) extensor carpi ulnaris release, (E) wedge osteotomy, (F) after resection, (G) compress the osteotomy site, $(\mathrm{H})$ insert screw, and (I) after screw insertion. 

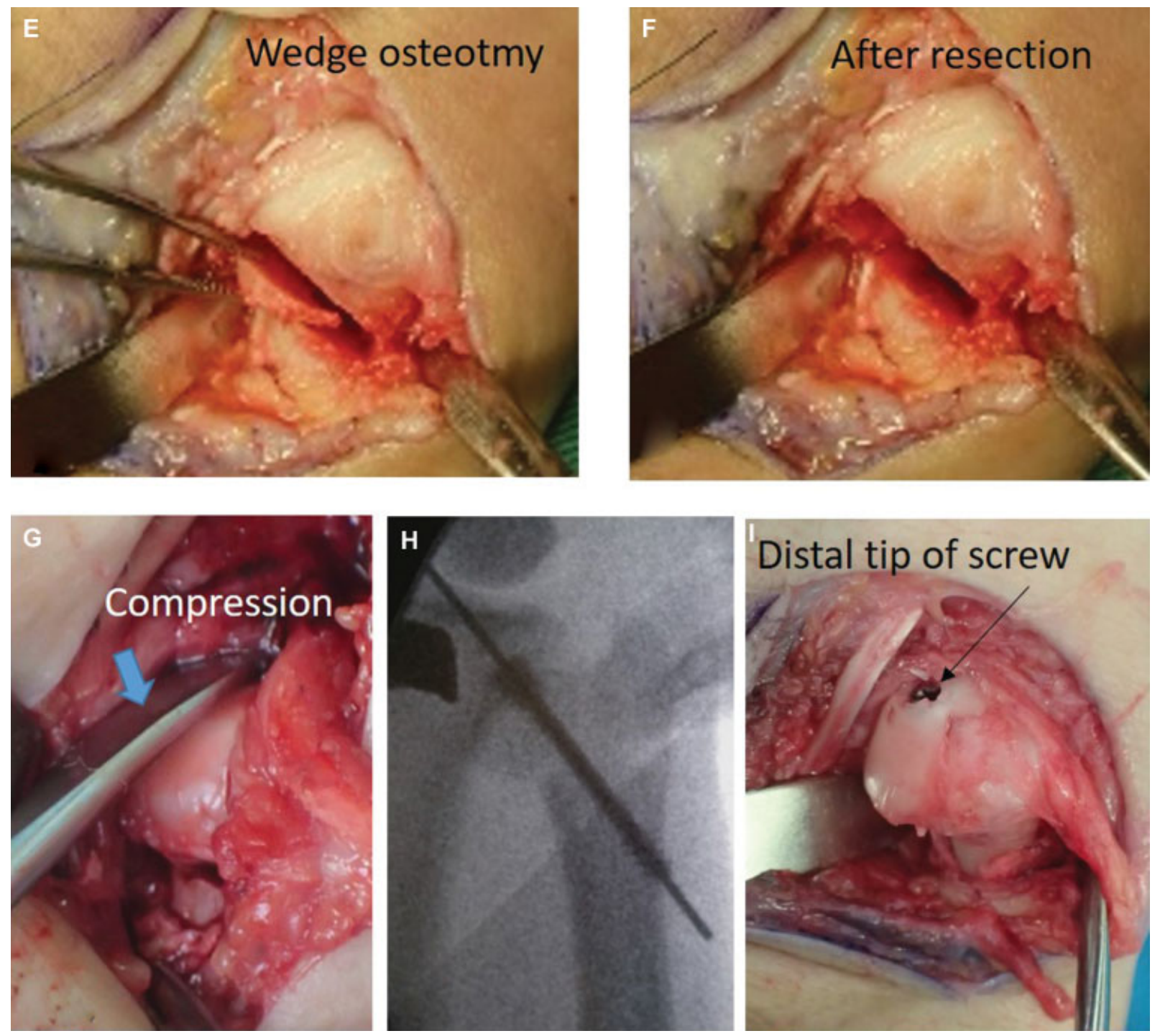

Fig. 2 (Continued)

the wrist until bone union. After bone union is confirmed, sports activity is allowed.

\section{Patients and Methods}

Distal ulnar metaphyseal wedge osteotomy with the modified Kitano's method was performed for 58 patients with ulnar abutment syndrome between 1995 and 2016. The clinical data of 43 (18 males, 25 females) of these patients who were followed for $>6$ months were analyzed. The indication for this procedure was ulnocarpal abutment syndrome with ulnar wrist pain especially in ulnar deviation which was not relieved by conservative treatment of $>6$ months. Cases with a foveal tear of the TFCC with instability of the DRUJ were excluded.

The mean age at operation was 43.2 years (16-75 years). The right wrist was affected in 26 patients, with the left wrist affected in 17 . They were diagnosed by physical examination, including the ulnocarpal stress test and shake hand test, which is our favorite physical examination for ulnar abut- ment syndrome. In this test, the examiner shakes hand with the patient and forces the patient's hand to ulnar deviation.

$\mathrm{X}$-ray examination of bilateral wrist in neutral position was performed for all patients and ulnar variance was measured. Ulnar variance is defined as the difference in length between the volar ulnar corner of the distal radius sigmoid notch and the most distal aspect of the dome of the ulnar head. Positive ulnar variance means that the dome of distal ulna is more distal than the ulnar corner of the distal radius. ${ }^{9}$ Cases with positive ulnar variance $<5 \mathrm{~mm}$ on X-ray are appropriate for this procedure. Cases with positive ulnar variance $>6 \mathrm{~mm}$ or with Madelung's deformity are excluded. The shape of sigmoid notch did not affect patient selection.

Magnetic resonance imaging was obtained in 17 cases: 7 cases showed high intensity area of the disc. Computed tomography arthrography ${ }^{14}$ of the DRUJ was obtained in 32 patients. Foveal tear of TFCC was not detected in any patients in these images.

In 29 patients, we investigated TFCC and articular surface by wrist arthroscopy before the osteotomy. Articular surface 


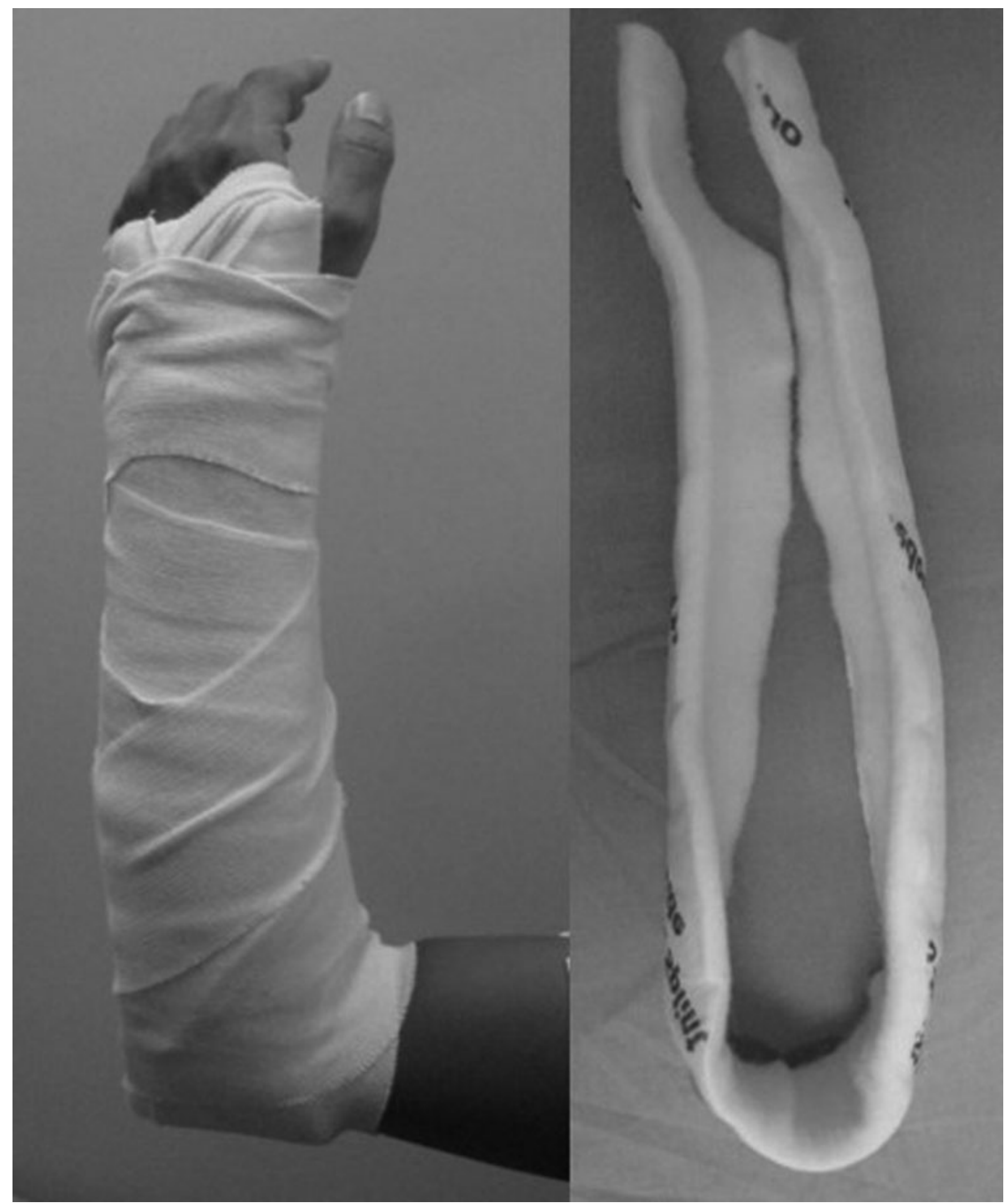

Fig. 3 Splint in the shape of sugar tongs.

fibrillation of lunate or ulnar head was observed in 28 patients. Perforation of TFCC was detected in 13 patients, but no foveal tear was detected in any patients.

Range of motion, grip strength, ulnar variance, and HAND20 were assessed before and after surgery. HAND20 is an original Japanese subjective scoring system for function that is easy to answer using a simple illustrated questionnaire. ${ }^{15}$ It has similar reliability and validity compared with Disabilities of the Arm, Shoulder and Hand (DASH).
The mean follow-up period was 21.9 months (range, 6-72 months).

\section{Results}

All patients experienced relief from preoperative ulnar wrist pain and returned to their work or sports activities. Bone union was achieved in all cases at an average of 2.6 months (1-6 months). Ulnar variance on X-ray of wrist decreased 
Table 1 Preoperative and postoperative examination data

\begin{tabular}{|l|l|l|l|}
\hline & Before & After & $p$-Value \\
\hline Dorsiflexion & 63.1 & 69.1 & $<0.05$ \\
\hline Palmar flexion & 60.9 & 63.8 & n.s. \\
\hline Pronation & 77 & 78.8 & n.s. \\
\hline Supination & 85 & 83.6 & n.s. \\
\hline Grip strength & 26.6 & 31.2 & $<0.05$ \\
\hline $\begin{array}{l}\text { Grip strength ratio to } \\
\text { unaffected hand }\end{array}$ & $77 \%$ & $87 \%$ & $<0.05$ \\
\hline HAND20 & 41.3 & 22.4 & $<0.05$ \\
\hline Ulnar variance & 2.2 & -0.6 & $<0.05$ \\
\hline
\end{tabular}

Abbreviation: n.s., not significant.

from $2.2 \mathrm{~mm}$ preoperatively to $0.6 \mathrm{~mm}$ postoperatively (-Table 1). Grip strength compared with the contralateral hand improved significantly from $77 \%$ preoperatively to $87 \%$ postoperatively $(p<0.05)$. The range of dorsiflexion improved significantly from $63.1^{\circ}$ preoperatively to $69.1^{\circ}$ postoperatively $(p<0.05)$. The range of palmar flexion, pronation, and supination changed from $60.9^{\circ}, 77.0^{\circ}$, and $85.0^{\circ}$ preoperatively to $63.8^{\circ}, 78.8^{\circ}$, and $83.6^{\circ}$ postoperatively, respectively. These differences in range of motion were not significant.

HAND20 at final follow-up improved significantly from 41.3 points preoperatively to 22.4 points postoperatively $(p<0.05)$. No patient showed osteoarthritis and related symptoms, while signs of remodeling of the ulnar head to match the DRUJ were found in 14 cases on X-ray at final follow-up.

\section{Complication}

In one case, prominence of the proximal tip of the screw caused irritation of the ECU tendon, and removal of the screw was necessary. After removal, this symptom disappeared. In three cases with remarkable osteoporosis, loss of continuity of the ulnar side cortex of the ulna at osteotomy was seen postoperatively, resulting in insufficient fixation of the distal fragment, and bony union delayed to 4.8 months in average (4 to 6 months). Following these experiences, when bone is osteoporotic and ulnar side cortex of the ulna is unstable, we add percutaneous pinning to secure the fixation that was removed 2 weeks postoperatively.

Ulnar styloid impingement occurred in one case, and ulnar styloid resection was performed for this patient 7 months after osteotomy.

\section{Case Presentation}

A 22-year-old professional golf player had left ulnar wrist pain after practice. He received conservative therapy from his local doctor for 1 month, but it failed to improve the symptom. Therefore, he came to our hospital. Preoperative physical examination showed a positive ulnocarpal stress test and no instability of the DRUJ. Preoperative ulnar variance was $+2 \mathrm{~mm}$ (-Fig. 4). He had a desire for a quick return to competition, so distal ulnar metaphyseal wedge osteotomy

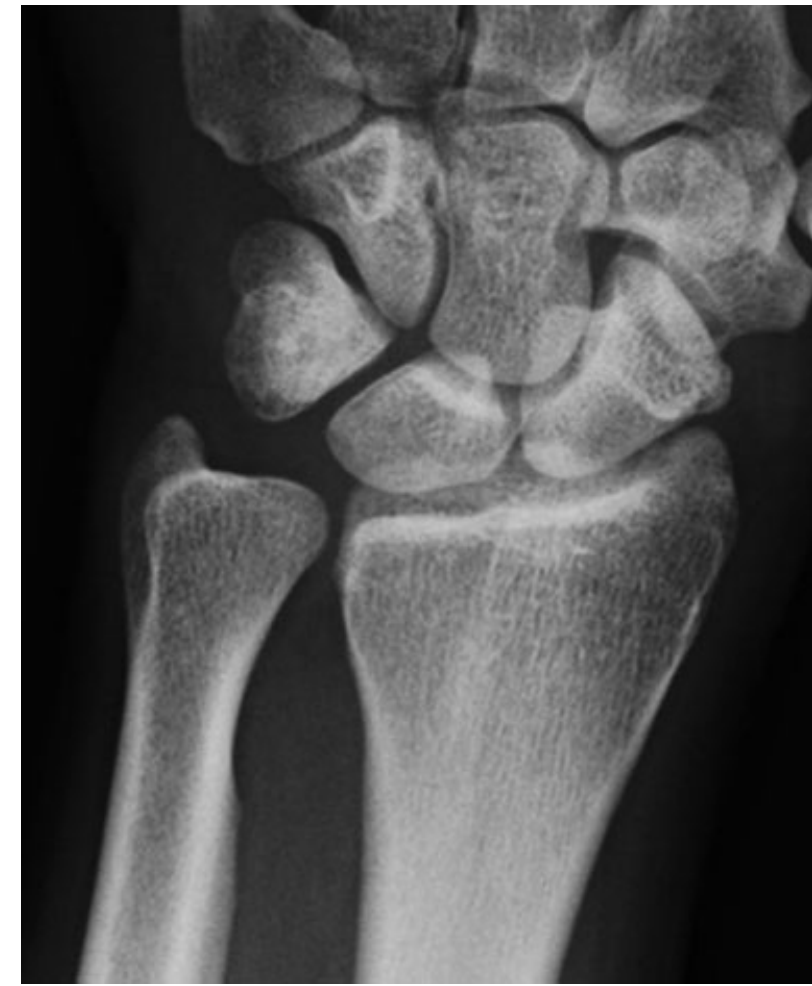

Fig. 4 Preoperative X-ray of the case.

was preferred to diaphyseal ulnar shortening osteotomy. Postoperative ulnar variance was $-2 \mathrm{~mm}$ ( - Fig. 5). After immobilization for 3 weeks, range of motion exercise was begun. Three months later, union was completed on X-ray examination (-Fig. 6), and he started to play golf again. Five months later, he returned to competition. The range of motion and grip strength did not change, but HAND20 was significantly improved from 43 points to 0 points.

\section{Discussion}

Diaphyseal ulnar shortening osteotomy is a widely accepted procedure, and many excellent results have been reported. ${ }^{2-9}$ One of the advantages of this procedure is to achieve stability of the DRUJ. ${ }^{16}$ On the other hand, as a disadvantage, it often takes $>5$ months for bony union, and delayed union and nonunion often occur, ${ }^{3}$ whereas recently better bone union was reported by using compression device and low profile plate system. ${ }^{17}$ Another disadvantage is that a relatively large skin incision is necessary for plate fixation, and plate removal is often necessary because of irritation to the ECU tendon.

Distal ulnar metaphyseal wedge osteotomy was first reported by Kitano et al in the Japanese literature in 1993, ${ }^{11}$ and Yoshida et al modified the Kitano method and reported better clinical results in 1998 in Japan. ${ }^{12}$ In 2007, Slade and Gillon first published distal ulnar metaphyseal wedge osteotomy in the English literature, which is similar to our technique, but no clinical results were described. ${ }^{18}$ Later, a few case reports with small number of patients of distal ulnar metaphyseal wedge osteotomy were reported. ${ }^{19-23}$ These reported a generally good results with early bone union. 


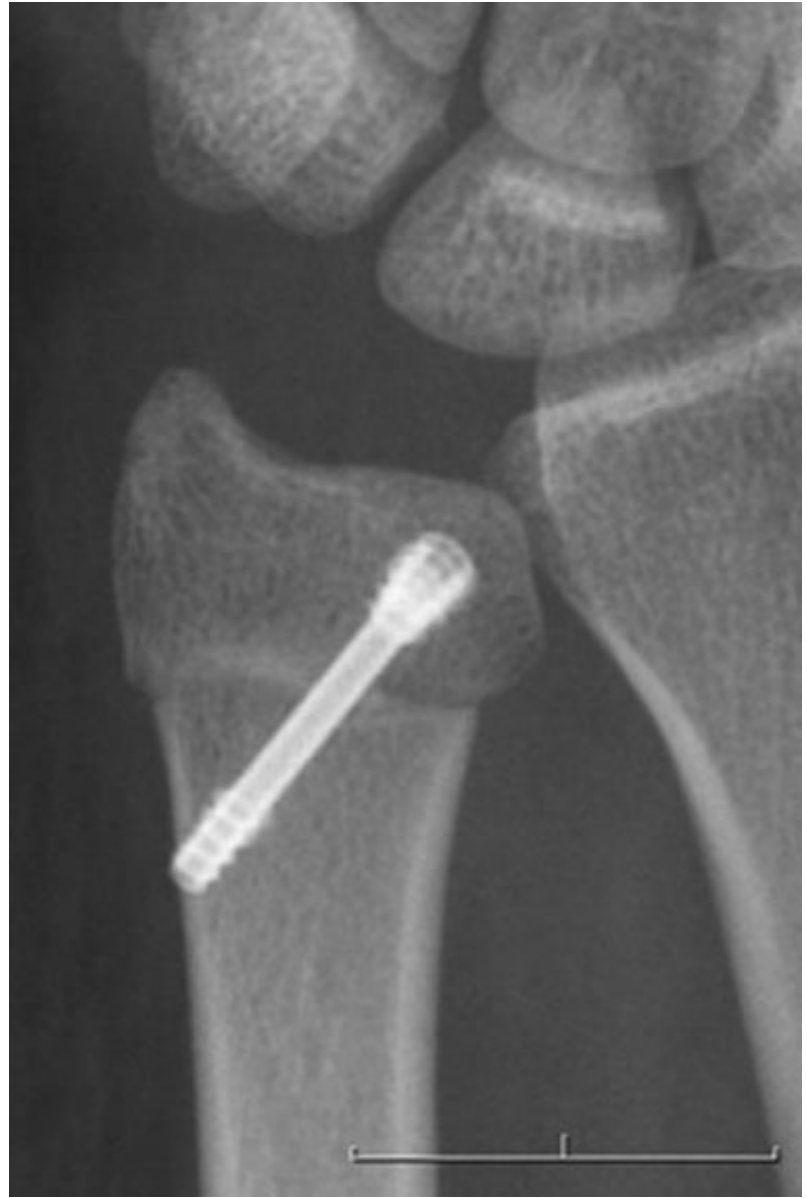

Fig. 5 X-ray at 1 month after operation of the case.

Distal ulnar metaphyseal wedge osteotomy has some advantages compared with diaphyseal ulnar shortening osteotomy with conventional technique. First, it can be performed with about a 3-cm skin incision and is less invasive. Second, a shorter union period (average 3 months) is expected because of the richer vascularity of the metaphyseal osteotomy site. Third, in principle, it is unnecessary to remove the headless screw, in contrast to plate fixation.

As a technical pearl of this procedure, the periosteal continuity of the ulnar aspect of the osteotomy site should be maintained for rigid fixation. If it is cut completely, the ulnar head becomes unstable. In such cases, we added percutaneous pinning for several weeks to secure fixation. As a disadvantage of the modified Kitano's method, the fovea shifts slightly in the radial direction, and the TFCC becomes slightly loose after this procedure. Thus, this procedure should not be used for cases that have instability of the DRUJ. Cases with positive ulnar variance $>6 \mathrm{~mm}$ or with Madelung's deformity are contraindications because decompression effect by rotation of the ulnar head may not be enough. Such cases are appropriate for ulnar shortening osteotomy of the diaphysis.

This study has some limitations. This was not a comparative study; therefore, the advantages in our method do not necessarily guarantee superiority to other procedures. This study included some cases with a short follow-up period,

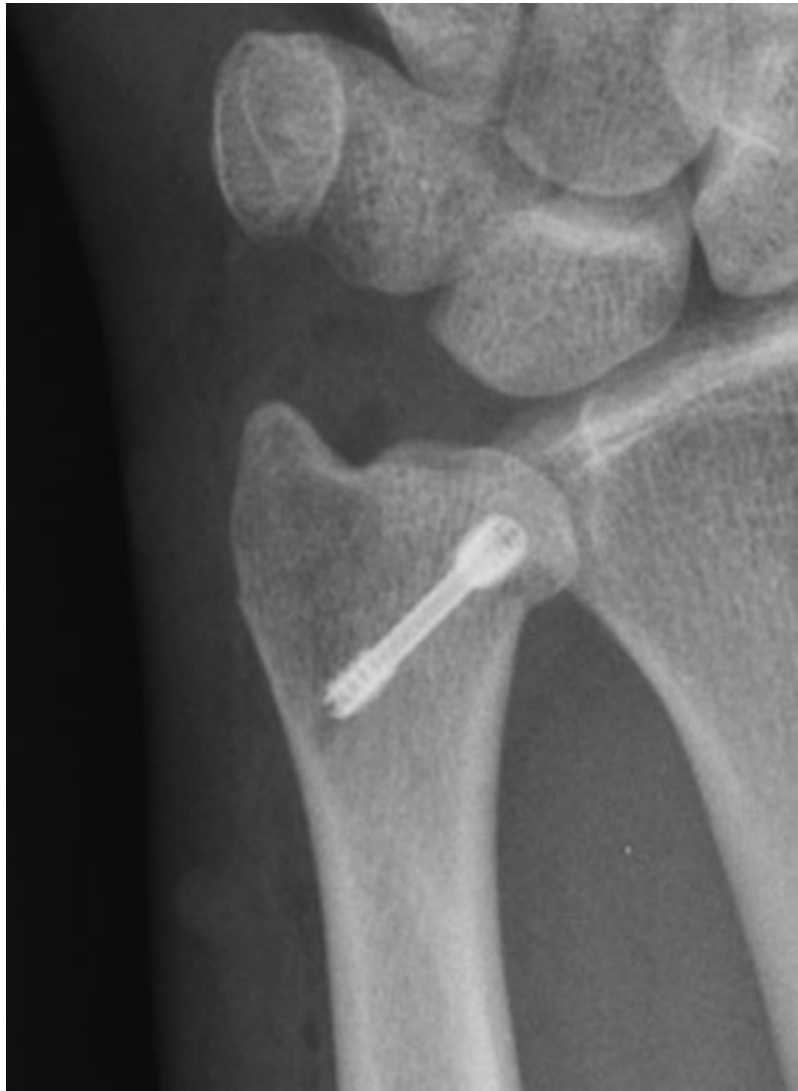

Fig. 6 X-ray at 3 months after operation of the case.

within 1 year; therefore, we need to review it with longer observation to see future osteoarthritic changes.

Distal ulnar metaphyseal wedge osteotomy was performed for patients with ulnocarpal abutment syndrome without DRUJ instability, and excellent outcomes were achieved. This procedure has several advantages compared with diaphyseal osteotomy, including minimal invasion and early bone union. This procedure should be considered one of the options to treat ulnar abutment syndrome.

Conflict of Interest

None declared.

\section{References}

1 Milch $\mathrm{H}$. Cuff resection of the ulna for malunited Colles' fracture. J Bone Joint Surg 1941;32A:311-313

2 Rayhack JM, Gasser SI, Latta LL, Ouellette EA, Milne EL. Precision oblique osteotomy for shortening of the ulna. J Hand Surg Am 1993;18(05):908-918

3 Köppel M, Hargreaves IC, Herbert TJ. Ulnar shortening osteotomy for ulnar carpal instability and ulnar carpal impaction. J Hand Surg Am 1997;22B:451-456

4 Darrow JC Jr, Linscheid RL, Dobyns JH, Mann JM III, Wood MB, Beckenbaugh RD. Distal ulnar recession for disorders of the distal radioulnar joint. J Hand Surg Am 1985;10(04):482-491

5 Darlis NA, Ferraz IC, Kaufmann RW, Sotereanos DG. Step-cut distal ulnar-shortening osteotomy. J Hand Surg Am 2005;30(05):943-948

6 Wehbé MA, Cautilli DA. Ulnar shortening using the AO small distractor. J Hand Surg Am 1995;20(06):959-964 
7 Chen NC, Wolfe SW. Ulna shortening osteotomy using a compression device. J Hand Surg Am 2003;28(01):88-93

8 Barbaric K, Rujevcan G, Labas M, Delimar D, Bicanic G. Ulnar shortening osteotomy after distal radius fracture malunion: review of literature. Open Orthop J 2015;9:98-106

9 Luria S, Lauder AJ, Trumble TE. Comparison of ulnar-shortening osteotomy with a new Trimed dynamic compression system versus the Synthes dynamic compression system: clinical study. J Hand Surg Am 2008;33(09):1493-1497

10 Feldon P, Terrono AL, Belsky MR. The "wafer" procedure. Partial distal ulnar resection. Clin Orthop Relat Res 1992;(275):124-129

11 Kitano K, Yoshida T, Uemura K, Tada K, Kawai H. Distal ulnar wedge osteotomy for ulnocarpal abutment syndrome (A preliminary report). J Jpn Soc Surg Hand 1993;10:20-23 (Article in Japanese)

12 Yoshida T, Moritomo H, Tada K. Closed wedge osteotomy of distal ulnar metaphysis for ulnocarpal abutment syndrome. J Jpn Soc Surg Hand 1998;15:198-201 (Article in Japanese)

13 Spinner M, Kaplan EB. Extensor carpi ulnaris. Its relationship to the stability of the distal radio-ulnar joint. Clin Orthop Relat Res 1970;68(68):124-129

14 Moritomo H, Arimitsu S, Kubo N, Masatomi T, Yukioka M. Computed tomography arthrography using a radial plane view for the detection of triangular fibrocartilage complex foveal tears. J Hand Surg Am 2015;40(02):245-251

15 Suzuki M, Kurimoto S, Shinohara T, Tatebe M, Imaeda T, Hirata H. Development and validation of an illustrated questionnaire to evaluate disabilities of the upper limb. J Bone Joint Surg Br 2010; 92(07):963-969
16 Arimitsu S, Moritomo H, Kitamura T, et al. The stabilizing effect of the distal interosseous membrane on the distal radioulnar joint in an ulnar shortening procedure: a biomechanical study. J Bone Joint Surg Am 2011;93(21):2022-2030

17 Finnigan T, Makaram N, Baumann A, Ramesh K, Mohil R, Srinivasan M. Outcomes of ulnar shortening for ulnar impaction syndrome using the $2.7 \mathrm{~mm}$ AO ulna shortening osteotomy system. J Hand Surg Asian Pac Vol 2018;23(01):82-89

18 Slade JF III, Gillon TJ. Osteochondral shortening osteotomy for the treatment of ulnar impaction syndrome: a new technique. Tech Hand Up Extrem Surg 2007;11(01):74-82

19 Khouri JS, Hammert WC. Distal metaphyseal ulnar shortening osteotomy: technique, pearls, and outcomes. J Wrist Surg 2014;3 (03):175-180

20 Hammert WC, Williams RB, Greenberg JA. Distal metaphyseal ulnar-shortening osteotomy: surgical technique. J Hand Surg Am 2012;37(05):1071-1077

21 Yin HW, Qiu YQ Shen YD, Xu JG, Gu YD, Xu WD. Arthroscopic distal metaphyseal ulnar shortening osteotomy for ulnar impaction syndrome: a different technique. J Hand Surg Am 2013;38 (11):2257-2262

22 Sennwald G, Della Santa D, Beaulieu JY. A comparison of diaphyseal and metaphyseal techniques of ulna shortening. J Hand Surg Eur Vol 2013;38(05):542-549

23 Nunez FA Jr, Barnwell J, Li Z, Nunez FA Sr. Metaphyseal ulnar shortening osteotomy for the treatment of ulnocarpal abutment syndrome using distal ulna hook plate: case series. J Hand Surg Am 2012;37(08):1574-1579 